Supporting Information

Constructing Phase-Transitional NiS $\mathbf{a}_{\mathbf{a}}$ Nitrogen-Doped Carbon Cathode Material with High Rate Capability and Cycling Stability for Alkaline

\title{
Zinc-Based Batteries
}

Nana Chang, t, Yanbin Yin, + Yang Song, ${ }^{\dagger}$ Shengnan Wang, t, Huamin Zhang, ${ }^{+}$Qinzhi

Lai, *, *,s Xianfeng Li*t

† Division of Energy Storage, Dalian National Laboratory for Clean Energy, Dalian

Institute of Chemical Physics, Chinese Academy of Sciences, 457 Zhongshan Road,

Dalian 116023, China

‡ University of Chinese Academy of Sciences, Beijing 100049, China

$\S$ Department of Chemistry, College of Sciences, Northeastern University, Shenyang

110819, China (Current Address)

Corresponding Author

Qinzhi Lai ${ }^{*,+, \delta}$ and Xianfeng $\mathrm{Li}^{*, \dagger}$ 
E-mail: qinzhi1226@163.com; lixianfeng@dicp.ac.cn 


\section{Experimental Section}

The preparation of Ni-DDTC precursor: The Ni-DDTC was prepared via a facile singlesource precursor method. Typically, $10 \mathrm{mmol}$ of nickel chloride hexahydrate $\left(\mathrm{NiCl}_{2} \cdot 6 \mathrm{H}_{2} \mathrm{O}\right)$

and $5 \mathrm{mmol}$ of sodium diethyldithiocarbamatre (Na-DDTC, $\left.\left(\mathrm{C}_{2} \mathrm{H}_{5}\right)_{2} \mathrm{NCSSNa} \cdot 3 \mathrm{H}_{2} \mathrm{O}\right)$ were respectively dissolved in $100 \mathrm{ml}$ deionized water, forming solution $\mathrm{A}$ and $\mathrm{B}$. Then, the solution A was slowly mixed with solution B with rapid stirring. Finally, the coprecipitation (denoted as Ni-DDTC) was collected by filtration, washed with water for several times, and dried at $70{ }^{\circ} \mathrm{C}$ overnight.

The synthesis of NiS @NC composite: The as-obtained Ni-DDTC powder was placed in a quartz crucible and then loaded within a tubular furnace. The power was heated to the targeted temperature $\left(500 / 700 / 900{ }^{\circ} \mathrm{C}\right)$ at a heating rate of $5{ }^{\circ} \mathrm{C} \mathrm{min}^{-1}$ and kept at this temperature for $2 \mathrm{~h}$ with an Ar flow. After cooling down to the room temperature, the final composites named as NiS $@$ NC-500,-700, and -900 were taken out and collected.

Materials characterization: The morphologies of $\mathrm{NiS}_{\mathrm{x}} @ \mathrm{NC}$ composites were obtained by

field-emission scanning electron microscopy (FE-SEM, JSM-7800F, Japan) and 
transmission electron microscopy (TEM, JEM-2100, Japan). The Powder X-ray diffraction (XRD) patterns were recorded with a $2 \theta$ range of 10 to $80^{\circ}$ using an X-ray diffractometer (D8 ADVANCE ECO; RIGAKU, Japan) equipped with Cu-Ka radiation source at $40 \mathrm{kV}$ and $40 \mathrm{~mA}$ and scan rate of $10^{\circ} \mathrm{min}^{-1}$. The chemical structure of NiS $@ \mathrm{NC}$ composites was examined by FT-IR spectrometer (Nicolet iS50, Thermofisher). Each spectrum was recorded at a rate of 48 scans with a resolution of $4 \mathrm{~cm}^{-1}$, collected from 600 to $4000 \mathrm{~cm}^{-1}$ in reflection mode. The Raman spectroscopy was recorded using Advantage 532 Raman spectrometer (NanoWizard Ultra Speed \& inVia Raman, Germany) equipped with a HeNe laser emitting at $532 \mathrm{~nm}$ in the range of $1000-2000 \mathrm{~cm}^{-1}$. The chemical states of composites were investigated by X-ray photoelectron spectroscopy (XPS, ESCALAB 250xi) with Al Ka radiation source. The binding energy was corrected using the $C$ 1s corelevel (taken at $284.8 \mathrm{eV}$ ) as the reference. The thermogravimetric analysis (TGA) of samples was conducted on a STA449F3 Thermal Analyzer in air with a temperature ranging from 50 to $600{ }^{\circ} \mathrm{C}$ at $5{ }^{\circ} \mathrm{C} \mathrm{min}-1$. The pore size distribution of $\mathrm{NiS}_{\mathrm{x}} @ \mathrm{NC}$ was analyzed by the Brunauer-Emmett-Teller surface area analyzer (ASAP 2020, Micromeritics). 
Electrochemical measurements: The cathode electrode was prepared by mixing NiS $@$ @NC with conductive agent of Super P carbon black, binder of polyvinylidene fluoride (PVDF) in a mass ratio of 8:1:1 and dissolved in N-methyl-pyrrolidone (NMP), forming a slurry. The obtained slurry was coated on a nickel foam (NF, size $3 \mathrm{~cm} \times 3 \mathrm{~cm})$ and then dried at $60{ }^{\circ} \mathrm{C}$ overnight. The $\mathrm{NiS}_{\mathrm{x}} @ \mathrm{NC}$ electrode was finally acquired by pressing the as-obtained square pieces at $10 \mathrm{MPa}$ for $60 \mathrm{~s}$. The mass loading of $\mathrm{NiS}_{\mathrm{x}} @ \mathrm{NC}$ active materials in NF electrode was about $10 \mathrm{mg} \mathrm{cm}^{-2}$. The electrochemical measurements were tested via a three-electrode system using $\mathrm{NiS}_{\mathrm{x}} @ \mathrm{NC}$ electrode as working electrode, graphite flake as counter electrode, $\mathrm{Hg} / \mathrm{HgO}$ electrode as reference electrode, and $2 \mathrm{M}$ $\mathrm{KOH}$ solution as electrolyte. Cyclic voltammetry (CV) test was conducted in a Reference 1000 electrochemical workstation (Gamry, USA). The CV spectra was investigated in a potential window from 0.25 to $0.55 \mathrm{~V}$ (vs. $\mathrm{Hg} / \mathrm{HgO}$ ). The capacitive effect was characterized by analyzing the cyclic voltammetry curves at various sweep rates according to the power-law equation:

$$
i=a v^{b}(1)
$$


Where $i\left(\mathrm{~A} \mathrm{~g}^{-1}\right)$ is the sum of the capacitive-controlled current $\left(i_{\mathrm{c}}\right)$ and diffusion-controlled current $\left(i_{\mathrm{d}}\right) ; v\left(\mathrm{mV} \mathrm{s}^{-1}\right)$ is the scan rate; the value of $\mathrm{b}$ is determined by the slope of linear $\log (\lambda)-\log (v)$. In general, if $b$-value is 0.5 , the energy storage process is controlled by diffusion, while 1.0 indicates a surface capacitance-controlled process. Hence, the calculated $\mathrm{b}$ value in this work is around 0.8 , which implies that the energy storage process is controlled by the diffusion and capacitive behavior synergistically. Specific contributions from each process can be quantified by the following equations:

$$
i(V)=i_{c}+i_{d}=k_{1} v+k_{2} v^{1 / 2}
$$

Equation (2) can be transformed to Equation (3):

$$
i(V) / v^{1 / 2}=k_{1} v^{1 / 2}+k_{2}
$$

According to Equation (3), the values of $k_{1}$ and $k_{2}$ can be acquired by reading the slope and intercept from the curves of linear $i / v^{1 / 2}-v^{1 / 2}$, and thereby both charge storage contributions are obtained though multiplying the k-value by $v$ and $v^{1 / 2}$ respectively. ${ }^{1}$ 
The electrochemical impedance spectroscopy (EIS) of the cathode electrode was measured by a $1260+1287$ electrochemical workstation (Solartron, England) and the applied frequency range was from $1000000 \mathrm{~Hz}$ to $0.1 \mathrm{~Hz}$ with an AC amplitude of $10 \mathrm{mV}$. The Zn//NiS $@$ @NC batteries (ZNBs) were assembled with $\mathrm{NiS}_{\mathrm{x}} @ \mathrm{NC}$ as cathode and zinc foil as anode in a self-designed square configuration (size $3 \mathrm{~cm} \times 2 \mathrm{~cm} \times 5 \mathrm{~cm}$ ). The distance between cathode and anode is $0.5 \mathrm{~cm}$. The The battery performance was performed by Neware (CT-4008-5V10A-FA, China) and Arbin (BT2000, China) battery charge/discharge systems. The $\mathrm{Zn} / / \mathrm{NiS}_{\mathrm{x}} @ \mathrm{NC}$ batteries were charged at different current densities ranging from 10 to $100 \mathrm{~mA} \mathrm{~cm}-2$ with a given charge capacity (15 mA h) and discharged to the cut-off voltage of $1.1 \mathrm{~V}$. To further demonstrate the scalability and feasibility of $\mathrm{NiS}_{\mathrm{x}} @ \mathrm{NC}$ composites, the $\mathrm{NiS}_{\mathrm{x}} @ \mathrm{NC}$ coated on carbon felt (NiS $\left.\mathrm{x}_{\mathrm{x}} @ \mathrm{NC}-\mathrm{CF}\right)$ cathode with charge capacity of 15 mA h was applied in Zn//NiS $@ @ N C$ flow batteries. This cathode was prepared by mixing $\mathrm{NiS}_{\mathrm{x}} @ \mathrm{NC}$ with binder of polyvinylidene fluoride (PVDF) in a mass ratio of 9: 1 and dissolved in dimethylacetamide (DMAC). The obtained slurry was coated on a carbon felt $(\mathrm{CF}$, size $3 \mathrm{~cm} \times 3 \mathrm{~cm})$ and then dried at $60^{\circ} \mathrm{C}$ overnight. 
The Zn//NiS $@ N C-C F$ battery was charged at $20 \mathrm{~mA} \mathrm{~cm}{ }^{-2}$ for 5 min and discharged to

$1.1 \mathrm{~V}$.
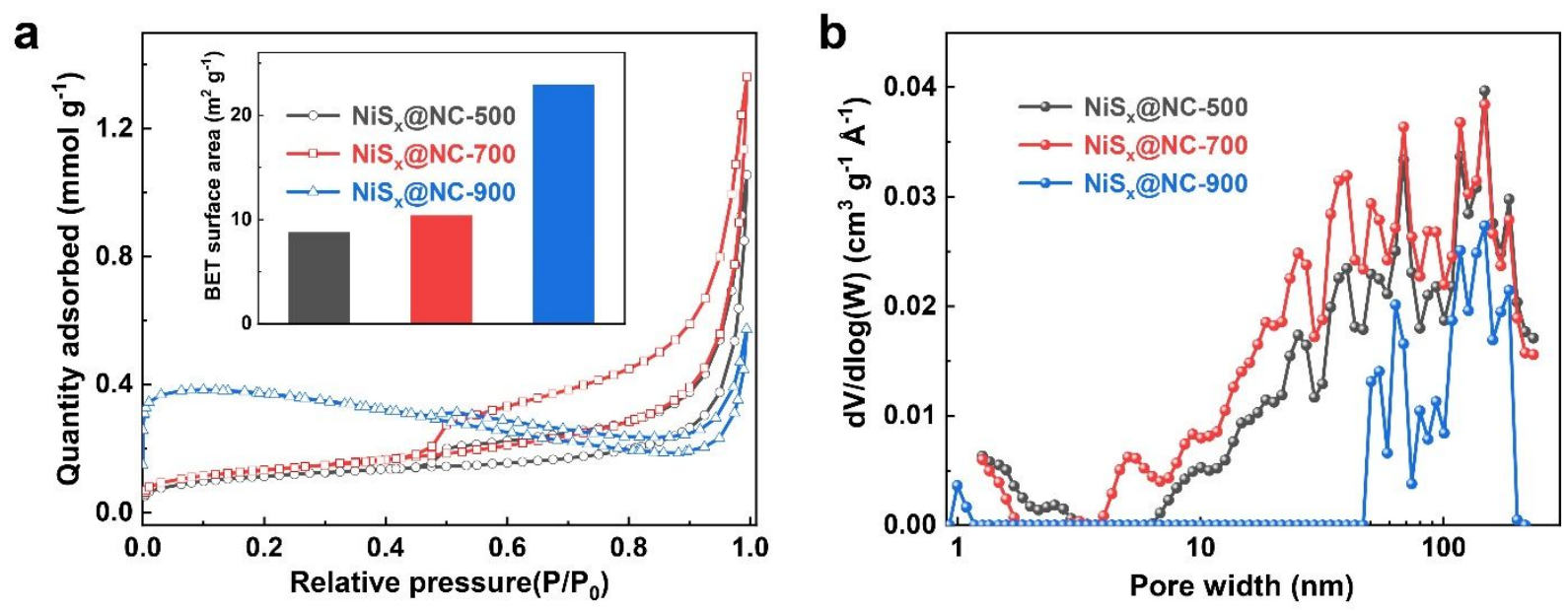

Figure S1. (a) Nitrogen adsorption-desorption isotherms and specific surface area (inset)

of $\mathrm{NiS}_{\mathrm{x}} @ \mathrm{NCs}$. (b) Pore size distributions of $\mathrm{NiS}_{\mathrm{x}} @ \mathrm{NCs}$.

The nitrogen adsorption-desorption isotherm was implemented to measure the surface area and porous structure of $\mathrm{NiS}_{\mathrm{x}} @ \mathrm{NC}-500,-700$, and -900 (Figure S1). The inset of Figure S1a clearly shows that the specific areas were increased from $8.8 \mathrm{~m}^{2} \mathrm{~g}^{-1}$ for $\mathrm{NiS}_{\mathrm{x}} @ \mathrm{NC}-500$ to $10.4 \mathrm{~m}^{2} \mathrm{~g}^{-1}$ for $\mathrm{NiS}_{\mathrm{x}} @ \mathrm{NC}-700$ and $22.9 \mathrm{~m}^{2} \mathrm{~g}^{-1}$ for $\mathrm{NiS}_{\mathrm{x}} @ \mathrm{NC}-900$ upon increasing the annealing temperature. In addition, both $\mathrm{NiS}_{\mathrm{x}} @ \mathrm{NC}-500$ and $\mathrm{NiS}_{\mathrm{x}} @ \mathrm{NC}-700$ display hierarchical porous structure with micropore, mesopore and macropore (Figure $\mathrm{S} 1 \mathrm{~b}$ ), which is beneficial for both electrochemical activity and ions transport. By contrast, $\mathrm{NiS}_{\mathrm{x}} @ \mathrm{NC}-900$ mainly includes micropore of size $1 \mathrm{~nm}$ and macropore, no mesopore at all. This could be ascribed to the fact that the pyrolysis rate of precursors at $900{ }^{\circ} \mathrm{C}$ is faster than that at lower temperatures $\left(500\right.$ and $\left.700{ }^{\circ} \mathrm{C}\right)$. Thus, all $\mathrm{NiS}_{\mathrm{x}} @$ NC-900 particles almost formed simultaneously and thereby more small particles was produced, which contributed to the formation of abundant micropores. However, these excessively small particles were not stable and they would inevitably aggregate at high temperature, which boosted the formation of macropores. By contrast, the $\mathrm{NiS}_{\mathrm{x}} @ \mathrm{NC}$ particles at lower temperatures $\left(500\right.$ and $700{ }^{\circ} \mathrm{C}$ ) formed slowly and thereby hierarchical porous structure including micropore, mesopore and macropore were achieved for $\mathrm{NiS}_{\mathrm{x}} @ \mathrm{NC}-500$ and -700. 


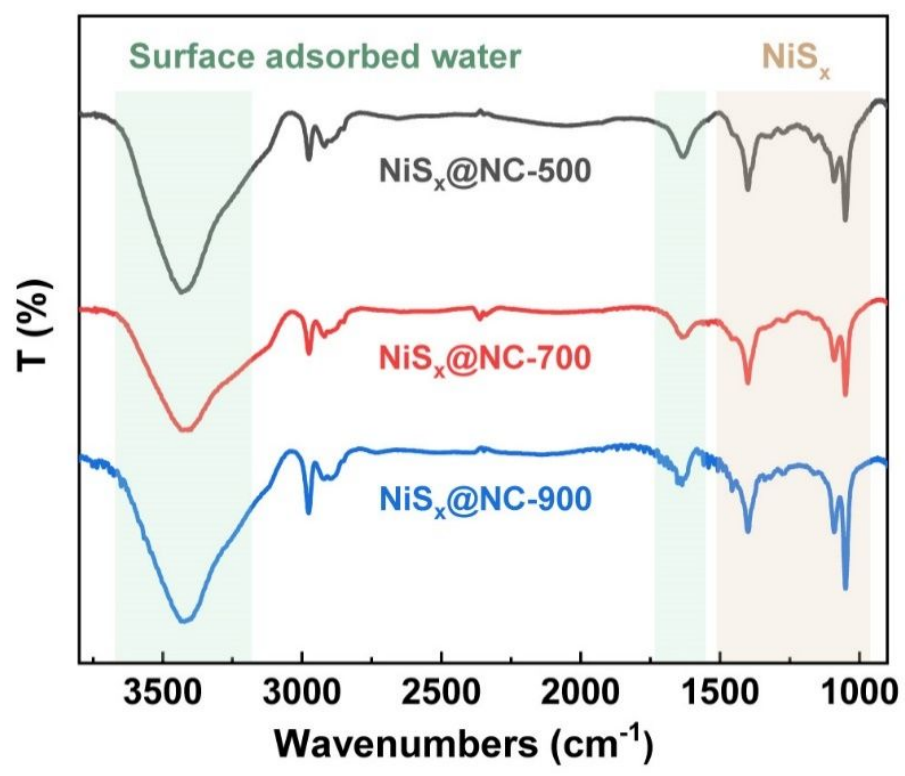

Figure S2. The FTIR spectra of $\mathrm{NiS}_{\mathrm{x}} @ \mathrm{NC}-500,-700$, and -900.

It should be noted that the peaks at $3300 \sim 3500$ and $1600 \sim 1650 \mathrm{~cm}^{-1}$ are assigned to the $\mathrm{OH}$ stretching and water bending vibrations stemming from the adsorbed water molecules on the surface of the samples.

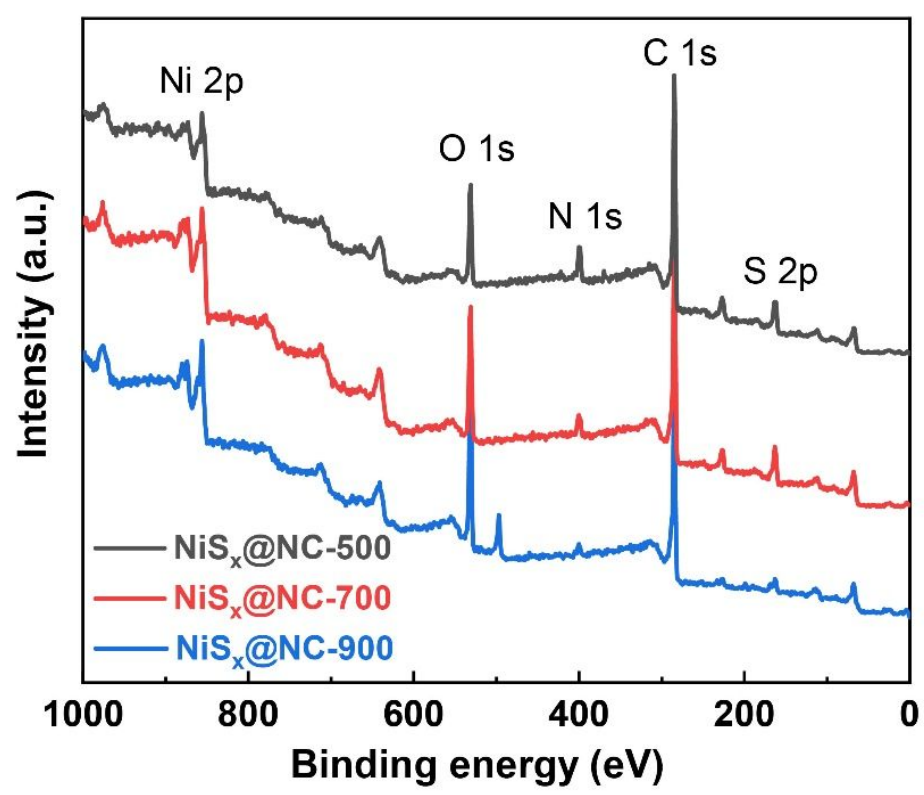

Figure S3. The full XPS spectra of $\mathrm{NiS}_{\mathrm{x}} @ \mathrm{NC}-500,-700$, and -900 . 
Table S1. Peak area of $2 \mathrm{p}_{3 / 2}$ energy band obtained from the fitting XPS spectra.

\begin{tabular}{|c|c|c|c|c|c|c|}
\hline Samples & $\mathbf{N i}^{\mathbf{0}}$ & $\mathrm{Ni}^{2+}$ & $\mathrm{Ni}^{0} / \mathrm{Ni}^{2+}$ ratio & $\mathbf{S}^{2-}$ & $\mathbf{S}_{2}{ }^{2-}$ & $\mathbf{S}^{2-} / \mathbf{S}_{2}{ }^{2-}$ ratio \\
\hline $\mathbf{N i S}_{\mathbf{x}} @ \mathbf{N C - 5 0 0}$ & 3363.1 & 11665.2 & 0.29 & 1209.2 & 2031.5 & 0.60 \\
\hline $\mathbf{N i S}_{\mathbf{x}} @ \mathbf{N C - 7 0 0}$ & 6415.3 & 16856.5 & 0.38 & 1297.3 & 1827.7 & 0.71 \\
\hline $\mathbf{N i S}_{\mathbf{x}} @ \mathbf{N C - 9 0 0}$ & 3207.6 & 14222.7 & 0.23 & 813.4 & 545.9 & 1.49 \\
\hline
\end{tabular}

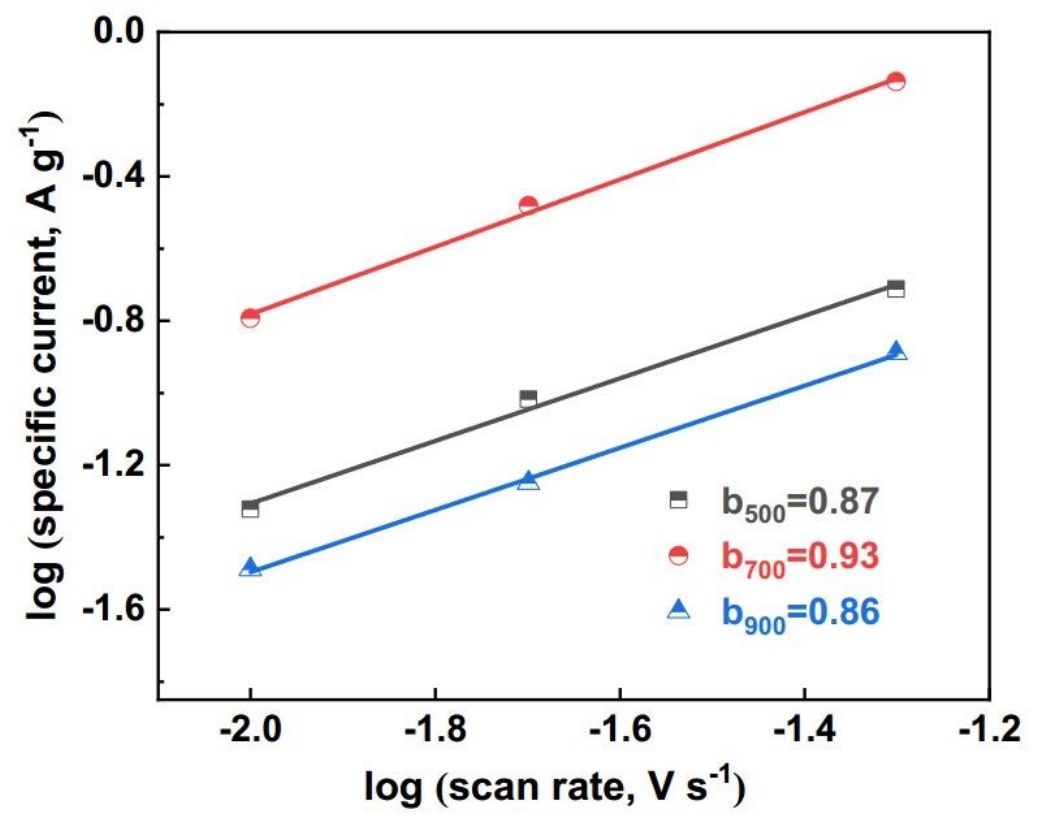

Figure S4. The fitting plot between $\log (i)$ and $\log (v)$ of $\mathrm{NiS}_{\mathrm{x}} @ \mathrm{NC}-500,-700$, and -900.

The $\mathrm{b}$ value could be determined by the slope of $\operatorname{linear} \log (i) \log (v)$, where the data is obtained from CV curves (Figure 3a-c). It should be noted that the data point at $5 \mathrm{mV} \mathrm{s}^{-1}$ was discarded in order to achieve higher goodness-of-fit. The detailed calculation process is on the basis of equation (1) (3) as described in experimental section. 


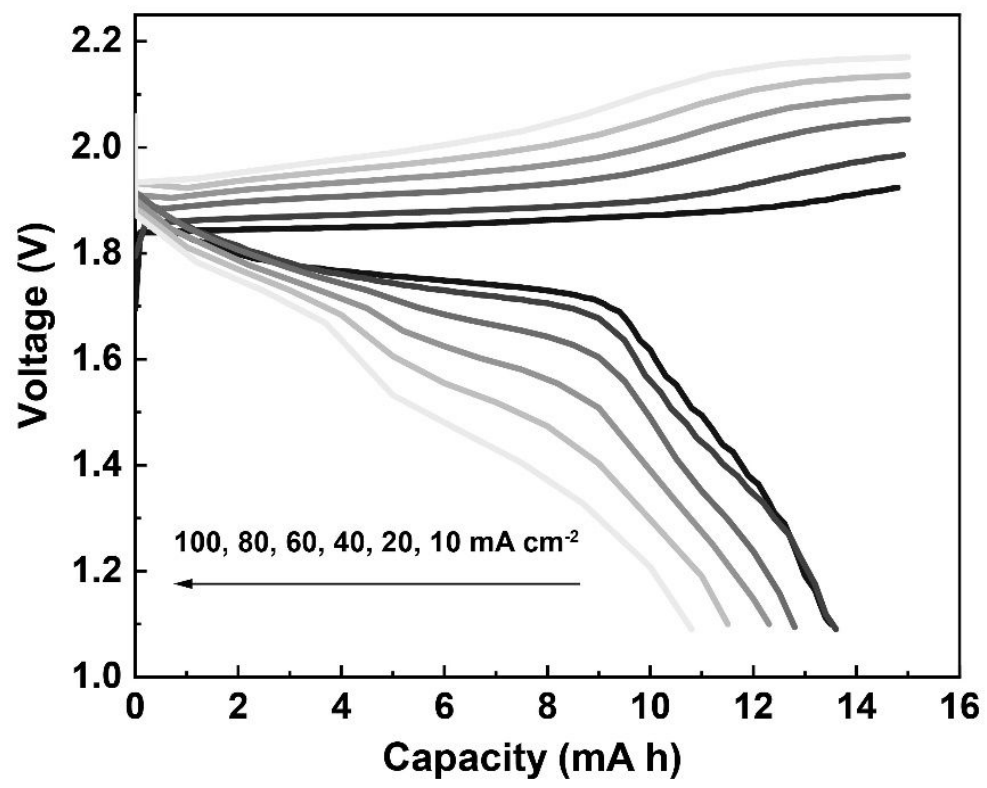

Figure S5. Rate performance of $\mathrm{NiS}_{\mathrm{x}} @ \mathrm{NC}-500$ at various current densities ranging from 10 to $100 \mathrm{~mA} \mathrm{~cm}^{-2}$.

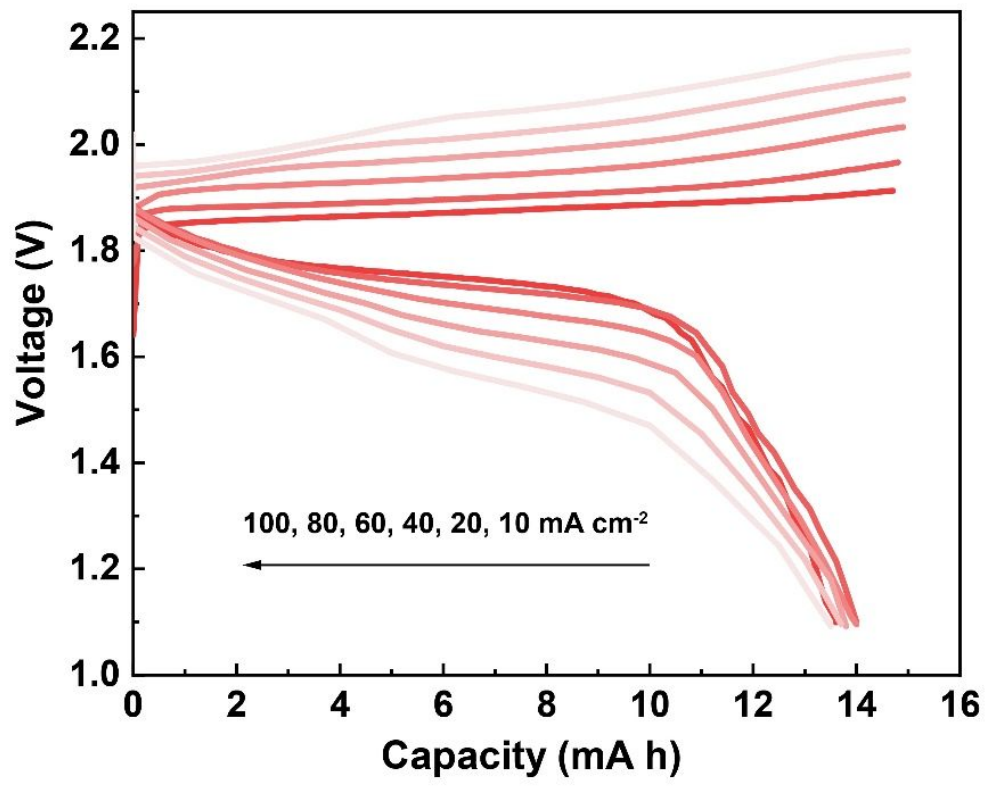

Figure S6. Rate performance of $\mathrm{NiS}_{\mathrm{x}} @ \mathrm{NC}-700$ at various current densities ranging from 10 to $100 \mathrm{~mA} \mathrm{~cm}^{-2}$. 


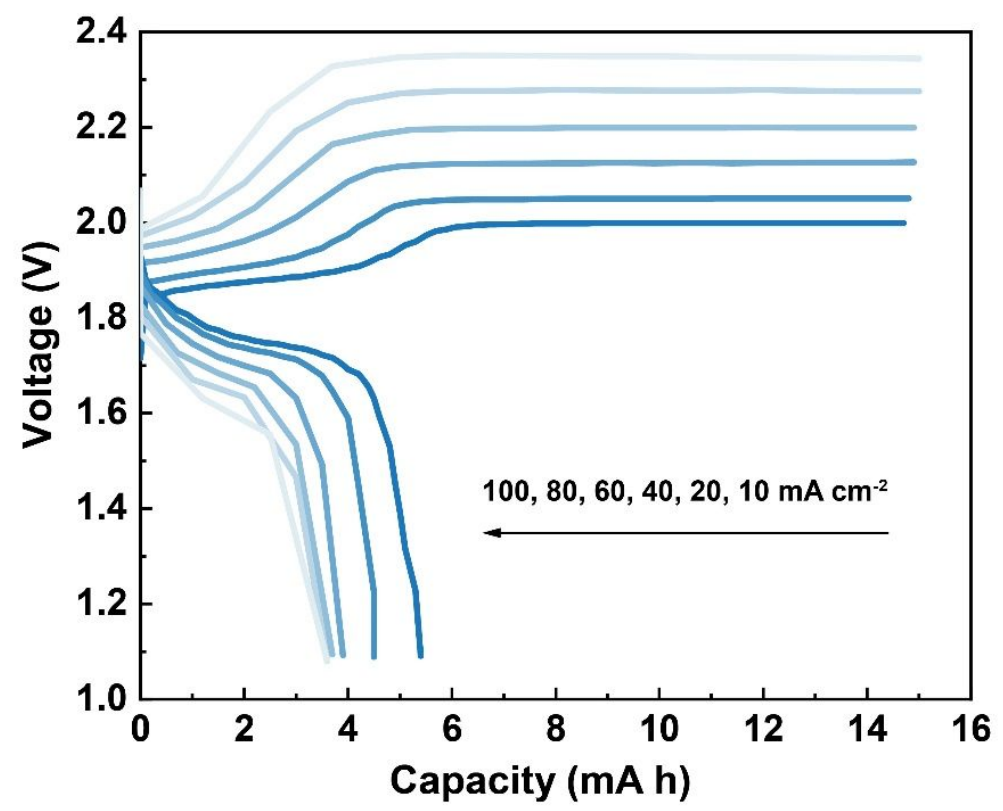

Figure S7. Rate performance of $\mathrm{NiS}_{\mathrm{x}} @ \mathrm{NC}-900$ at various current densities ranging from 10 to $100 \mathrm{~mA} \mathrm{~cm}^{-2}$.

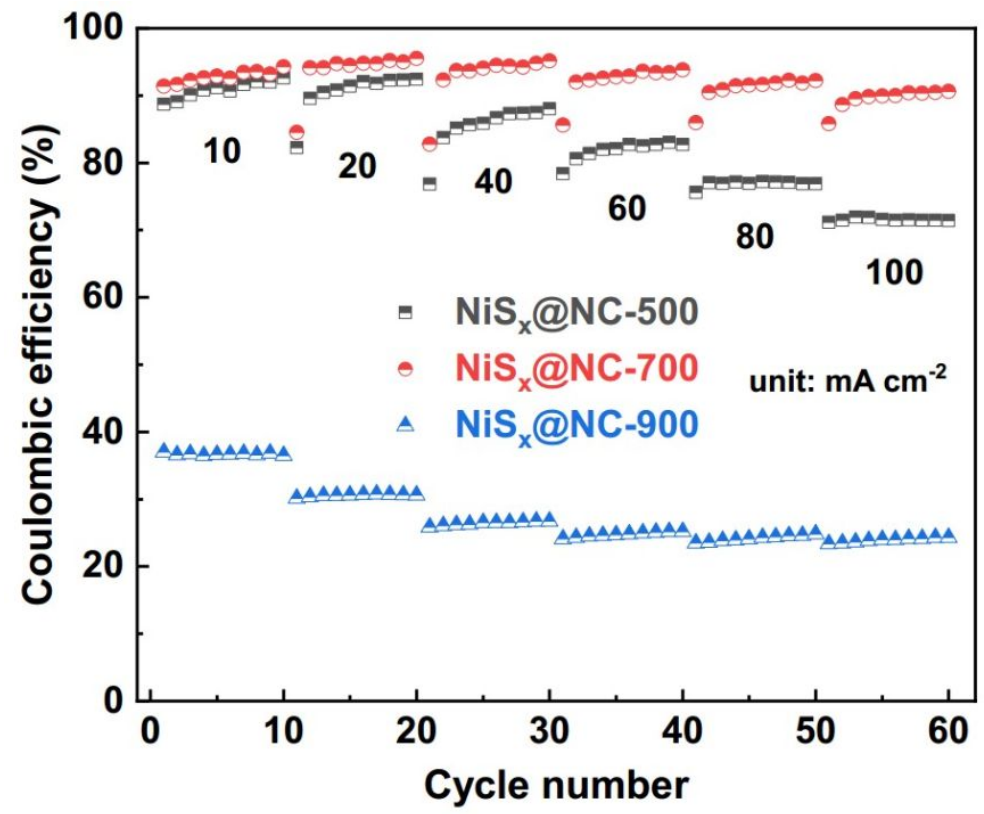

Figure S8. Coulombic efficiency (CE) of $\mathrm{NiS}_{\mathrm{x}} @ \mathrm{NC}-500,-700$, and -900 at various current densities ranging from 10 to $100 \mathrm{~mA} \mathrm{~cm}^{-2}$. 


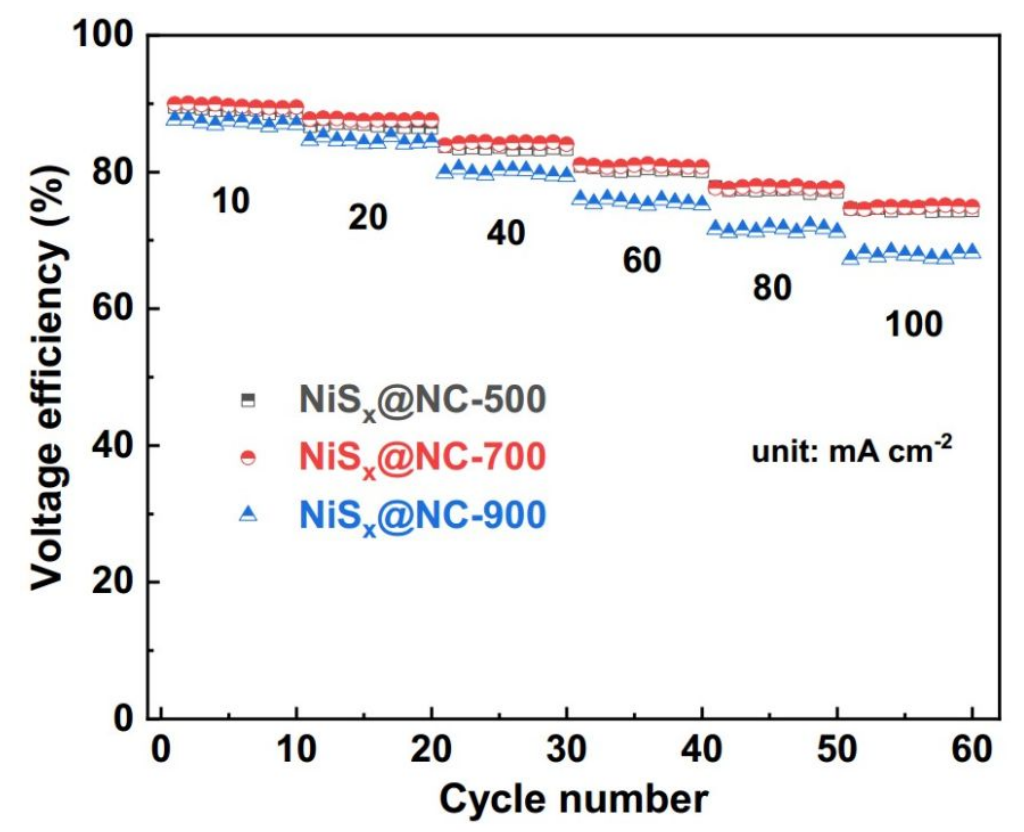

Figure S9. Voltage efficiency (VE) of $\mathrm{NiS}_{\mathrm{x}} @ \mathrm{NC}-500,-700$, and -900 at various current densities ranging from 10 to $100 \mathrm{~mA} \mathrm{~cm}^{-2}$.

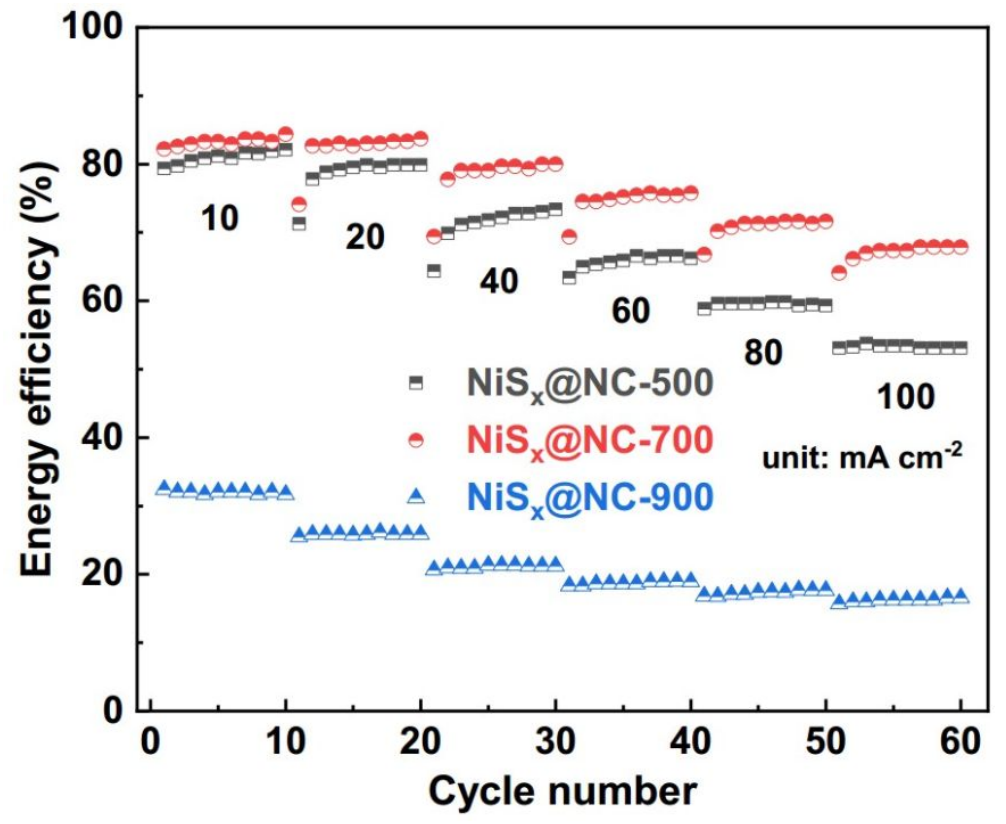

Figure S10. Energy efficiency $(\mathrm{EE})$ of $\mathrm{NiS}_{\mathrm{x}} @ \mathrm{NC}-500,-700$, and -900 at various current densities ranging from 10 to $100 \mathrm{~mA} \mathrm{~cm}^{-2}$. 


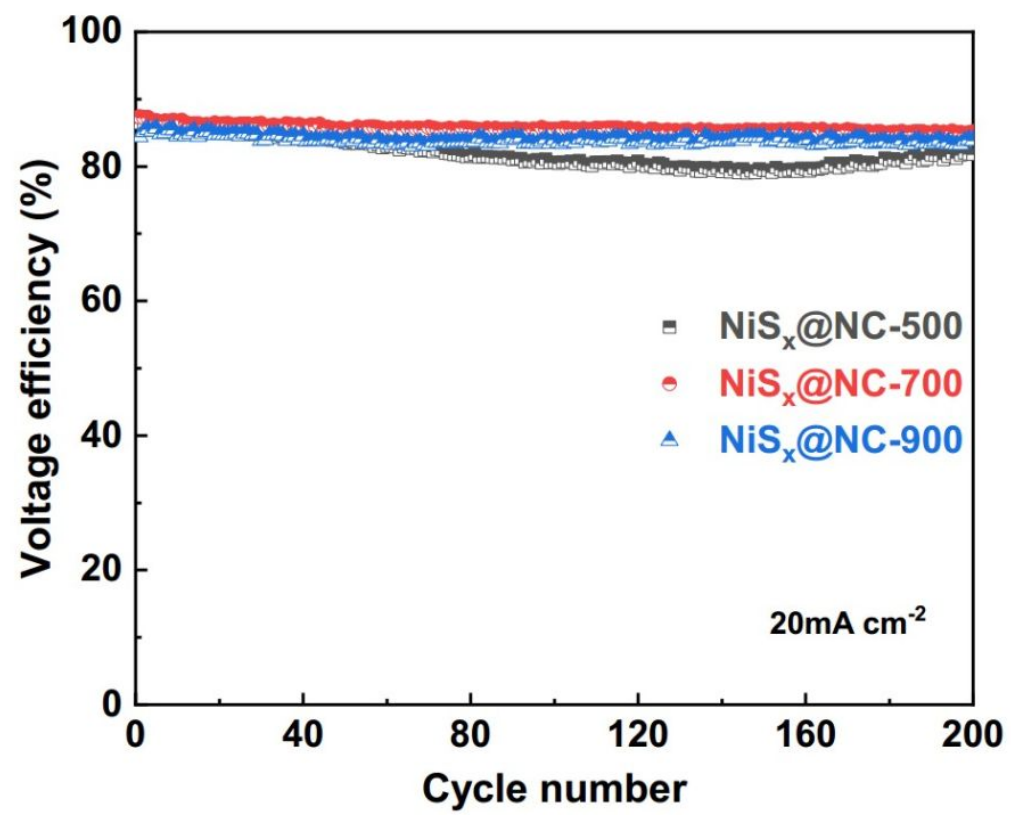

Figure S11. Cycling stability of voltage efficiency (VE) for the ZNBs with $\mathrm{NiS}_{\mathrm{x}} @ \mathrm{NC}$ at $20 \mathrm{~mA}$ $\mathrm{cm}^{-2}$.

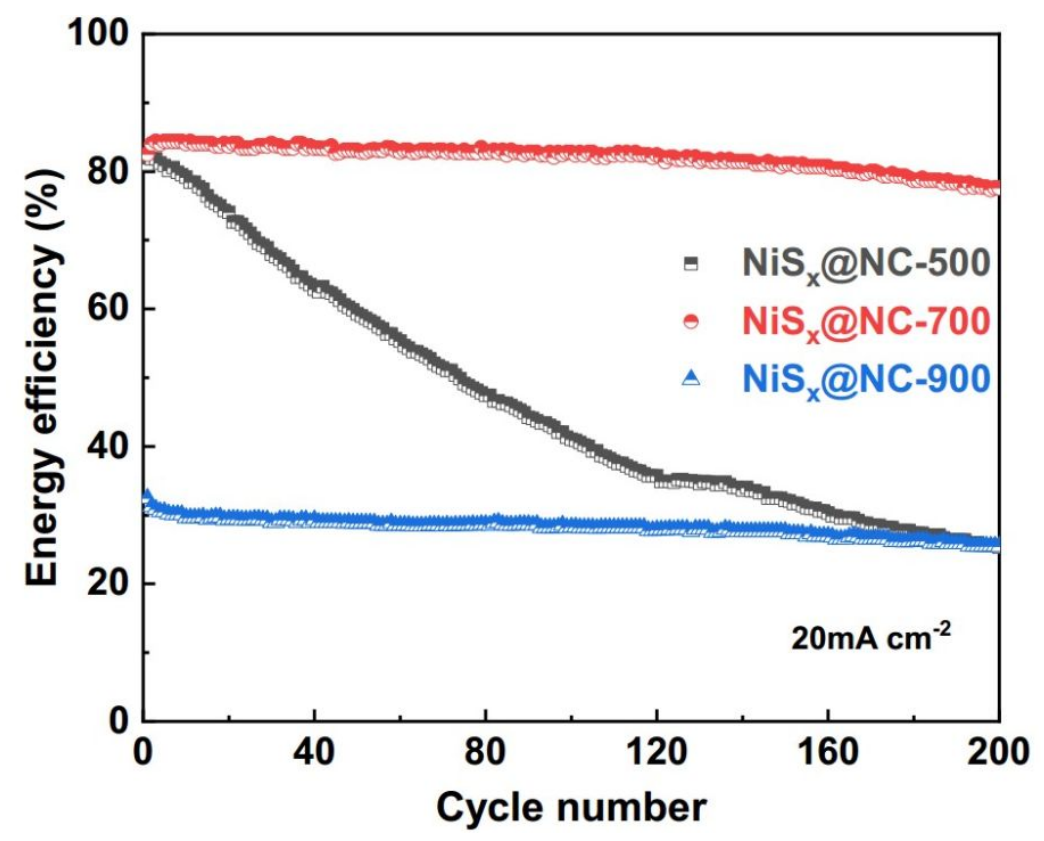

Figure S12. Cycling stability of energy efficiency (EE) for the ZNBs with $\mathrm{NiS}_{\mathrm{x}} @ \mathrm{NC}$ at $20 \mathrm{~mA}$ $\mathrm{cm}^{-2}$. 


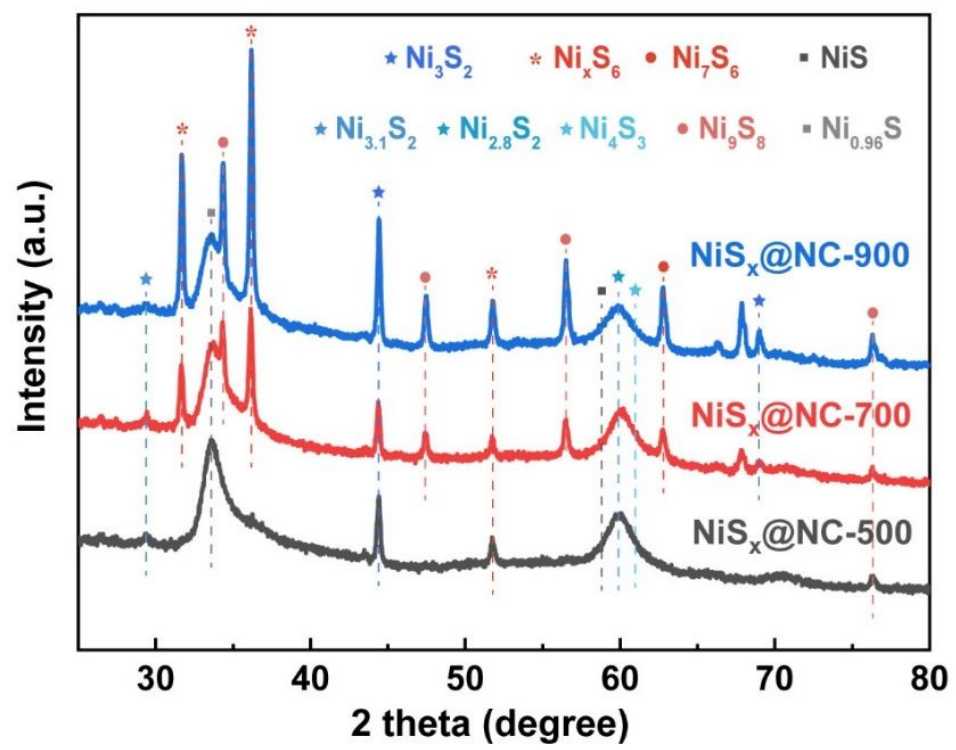

Figure S13. XRD patterns of NiS $@ N C-500,-700$, and -900 after 200 cycles.

The XRD patterns of $\mathrm{NiS}_{\mathrm{x}} @ \mathrm{NCs}$ after 200 cycles (Figure S13) show that the crystal structure of three kinds of $\mathrm{NiS}_{\mathrm{x}}$ have changed after cycling at different levels, which mainly includes some or all of the following mixed crystal forms with $\mathrm{Ni} / \mathrm{S}$ ratios ranging from 0.96 to $1.5\left(\mathrm{Ni}_{3} \mathrm{~S}_{2}, \mathrm{Ni}_{3.1} \mathrm{~S}_{2}\right.$, $\left.\mathrm{Ni}_{2.8} \mathrm{~S}_{2}, \mathrm{Ni}_{4} \mathrm{~S}_{3}, \mathrm{Ni}_{\mathrm{x}} \mathrm{S}_{6}, \mathrm{Ni}_{7} \mathrm{~S}_{6}, \mathrm{Ni}_{9} \mathrm{~S}_{8}, \mathrm{NiS}, \mathrm{Ni}_{0.96} \mathrm{~S}\right)$. The result indicates that pure phase of $\mathrm{NiS}$ $\left(\mathrm{NiS}_{\mathrm{x}} @ \mathrm{NC}-500\right)$ and $\mathrm{Ni}_{3} \mathrm{~S}_{2}\left(\mathrm{NiS}_{\mathrm{x}} @ \mathrm{NC}-900\right)$ will gradually transform into mixed phases, exactly as similar with transitional phase of $\mathrm{NiS}_{\mathrm{x}} @ \mathrm{NC}-700$, with cycles proceeding. This phenomenon demonstrates that multi-phase $\mathrm{NiS}_{\mathrm{x}}$ is more stable than single-phase $\mathrm{NiS}_{\mathrm{x}}$ during charge-discharge process. 

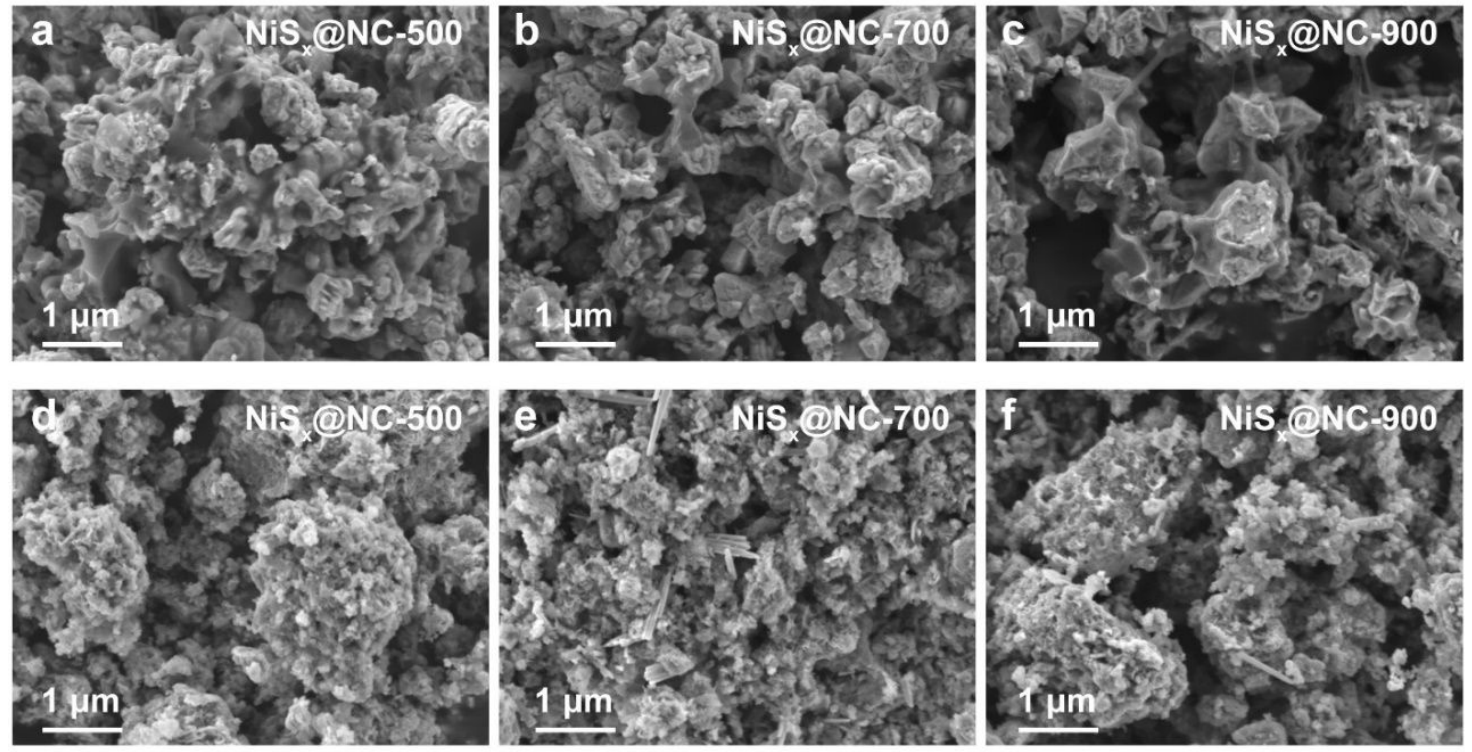

Figure S14. SEM images of NiS $\mathrm{x}_{\mathrm{x}} @ \mathrm{NC}-500,-700$, and -900 (a-c) before and (d-f) after 200 cycles.

From the SEM images of $\mathrm{NiS}_{\mathrm{x}} @ \mathrm{NCs}$ (Figure S14), we can see that there are small volumetric changes in microstructure of $\mathrm{NiS}_{\mathrm{x}} @ \mathrm{NC}-700$ after 200 cycles (Figure S14e) except for the disturbance from additional conductive carbon microspheres. This could be mainly ascribed to the slight crystal-structure changes of transitional phase $\mathrm{NiS}_{\mathrm{x}}$. However, agglomerated large particles are observed on the morphologies of $\mathrm{NiS}_{\mathrm{x}} @ \mathrm{NC}-500$ and -900 after cycling, which arises from large crystal-structure changes of pure-phase $\mathrm{NiS}_{\mathrm{x}}$. In addition, the better protective effect of the moderate-thickness NC layer further makes for the stable structure of $\mathrm{NiS}_{\mathrm{x}} @ \mathrm{NC}-700$.

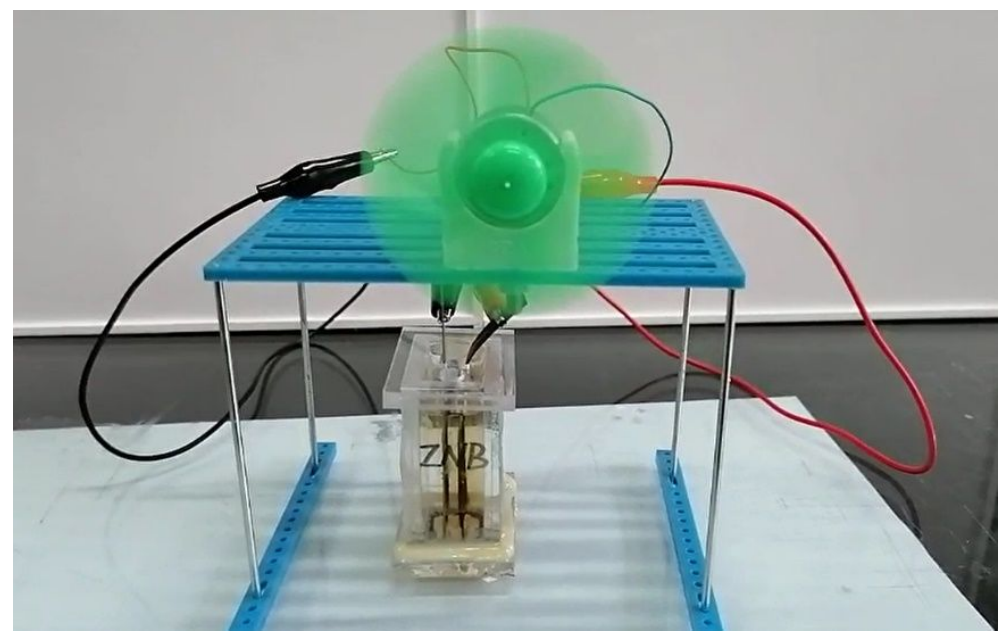


Figure S15. Photograph of an electric fan powered by a Zn// NiS $@$ @NC-700 battery.

\section{REFERENCES}

(1) J. Wang, J. Polleux, J. Lim and B. Dunn. Pseudocapacitive Contributions to Electrochemical

Energy Storage in $\mathrm{TiO}_{2}$ (Anatase) Nanoparticles. J. Phys. Chem. C 2007, 111, 14925-14931. 